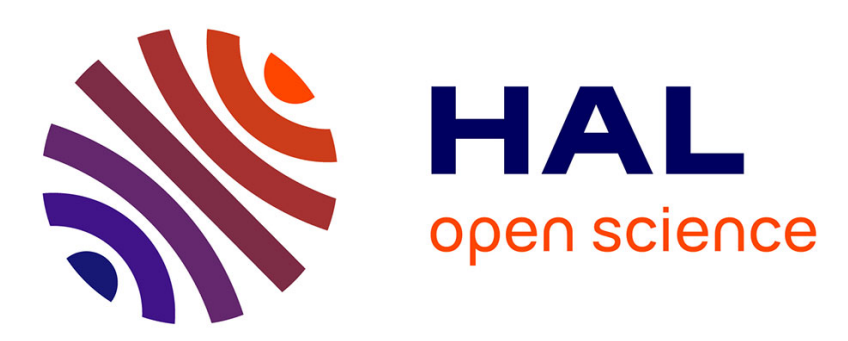

\title{
Two-phase Couette-Taylor flow: Arrangement of the dispersed phase and effects on the flow structures
}

\author{
Henda Djeridi, Céline Gabillet, Jean-Yves Billard
}

\section{To cite this version:}

Henda Djeridi, Céline Gabillet, Jean-Yves Billard. Two-phase Couette-Taylor flow: Arrangement of the dispersed phase and effects on the flow structures. Physics of Fluids, 2004, 16 (1), pp.128-139. 10.1063/1.1630323 . hal-01208349

\section{HAL Id: hal-01208349 \\ https://hal.science/hal-01208349}

Submitted on 2 Oct 2015

HAL is a multi-disciplinary open access archive for the deposit and dissemination of scientific research documents, whether they are published or not. The documents may come from teaching and research institutions in France or abroad, or from public or private research centers.
L'archive ouverte pluridisciplinaire HAL, est destinée au dépôt et à la diffusion de documents scientifiques de niveau recherche, publiés ou non, émanant des établissements d'enseignement et de recherche français ou étrangers, des laboratoires publics ou privés.

\section{(ㄷ)(1) $\$$}

Distributed under a Creative Commons Attribution - NonCommerciall 4.0 International 


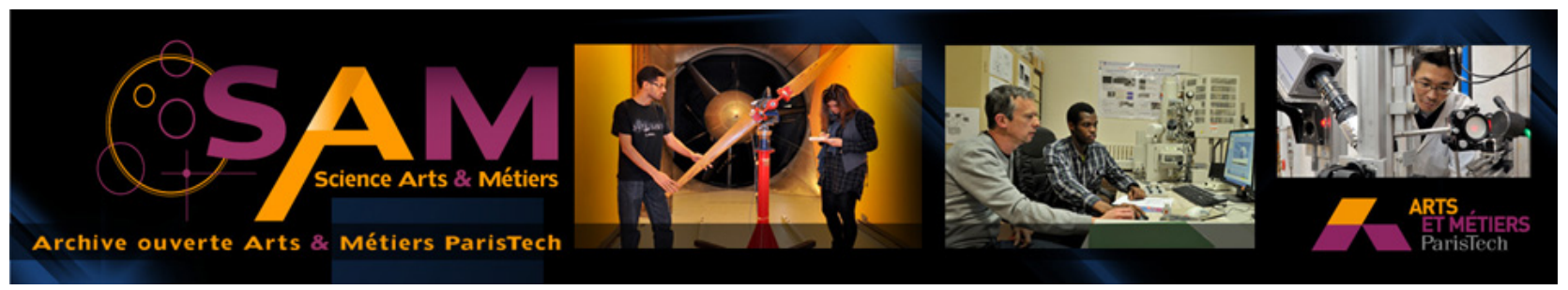

\section{Science Arts \& Métiers (SAM)}

is an open access repository that collects the work of Arts et Métiers ParisTech researchers and makes it freely available over the web where possible.

This is an author-deposited version published in: http://sam.ensam.eu

Handle ID: .http://hdl.handle.net/10985/10302

\section{To cite this version :}

Henda DJERIDI, Céline GABILLET, Jean-Yves BILLARD - Two-phase Couette-Taylor flow: Arrangement of the dispersed phase and effects on the flow structures - Physics of Fluids - Vol. $16, n^{\circ} 1$, p.128-139- 2004 


\title{
Two-phase Couette-Taylor flow: Arrangement of the dispersed phase and effects on the flow structures
}

\author{
H. Djeridi, C. Gabillet, and J. Y. Billard \\ IRENav, Institut de Recherche de l'Ecole Navaie, Lanvioc Poulmic, 29240 Brest Naval, France
}

\begin{abstract}
This study investigates the mutual interactions between a contimuous and a dispersed phase (noncondensable or condensable) in the well-known Couette-Taylor flow between two concentric cylinders at low Reynolds numbers, where the outer cylinder is immobilized In this experiment, the turbulent structures take place progressively. The noncondensable dispersed phase (air) is introduced either by ventilation, generated by agitation of a free surface situated at the top of the gap between the two cylinders. The condensable dispersed phase is generated by cavitation due to a drop in pressure. Comparisons are made between the single phase flow patterns and those observed in ventilated or cavitating flow. Two particular arrangements of the dispersed phase are experimentally evident, according to the Reynolds number of the flow. For low Reynolds numbers, bubbles are trapped in the core of the Taylor cells, whereas they migrate to the outflow regions near the inner cylinder for higher Reynolds numbers. Assessment of the forces applied to the bubbles and computation of their equilibrium position can act as a base in describing the bubble capture. When bubbles are located near the wall in the outflow region, it is found that the three first instabilities are strongly influenced by the dispersed phase. The cavitating flow is also characterized by an earlier appearance of the third instability.
\end{abstract}

\section{INTRODUCTION}

For many years, bubbly flows have received a great deal of attention in order to achieve a better understanding of the fundamental mechanisms of mutual interactions between the dispersed phase and the liquid flow structure. This is the case in many practical situations, such as the diffusion and transport process in centrifugal extractors or petroleum engineering systems.

Due to the extreme complexity of bubbly turbulent flows, many authors have become interested in studying the behavior of noncondensable bubbles in a vortex arrangement. Particular emphasis has been placed on the role of the coherent structures on transport and the capture of the

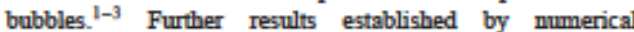
simulation ${ }^{4}$ showed the modifications induced by various sized bubbles on a single vortex in a vertical plane mixing layer.

The interactions between bubbles and vortex arrangements have been experimentally analyzed in the academic Couette-Taylor flow. Shiomi ot al..$^{5}$ performed measure ments in a concentric anmulus for turbulent regimes. The bubbly flow was obtained by the injection of a gas-liquid mixture at the bottom of the apparatus. In the aforementioned study, interest was focused on the correlation between the volumetric fluxes and the rotational speed of the inner cylinder, describing three main flow patterns: dispersed bubbly flow, ring form and spiral flow. However, the effects of the dispersed phase on the turbulence characteristics of the Conette-Taylor flow were not investigated without axial fluxes. Atkhen of al. ${ }^{6}$ analyzed the properties of the two- phase air-water turbulent Couette-Taylor flow. They clearly showed the particular features of this flow and described how air bubbles place themselves within the vortex patterns. They concluded that air bubbles remain situated along the outflow streamlines and represent a good tracer in order to describe the high Reynolds flow. Nevertheless, in their experiment, the superimposed axial flow and the agitation of the upper free surface limits the interpretation of the results, and particularly unclear is the effect of the dispersed phase on the turbulent flow structures. For smaller Reynolds numbers in the wavy vortex flow (WVF) regime, Djéridi et al. ${ }^{7}$ conducted experiments in a ventilated Couette-Taylor flow. Bubbles, generated by overturning breaking waves on the free surface at the top of the gap, are rapidly driven from the upper to the deepest cells of the apparatus, despite the buoyancy forces. For very low Reynolds numbers, preliminary results show that there is no sensitive effect of the noncondensable dispersed phase on the liquid flow patterns. These results give rise to two questions:

(i) Are there any effects on the Couette-Taylor flow patterms, due to the introduction of a noncondensable dispersed phase, for larger Reynolds numbers?

(ii) For the same range of Reynolds numbers, what similarities, if any, are to be found between noncondensable and condensable dispersed phase effects on these flow patterns?

A two-phase Couette-Taylor flow is experimentally investigated in this paper, with either air bubbles generated by ventilation, or vapor bubbles generated by cavitation. In the 


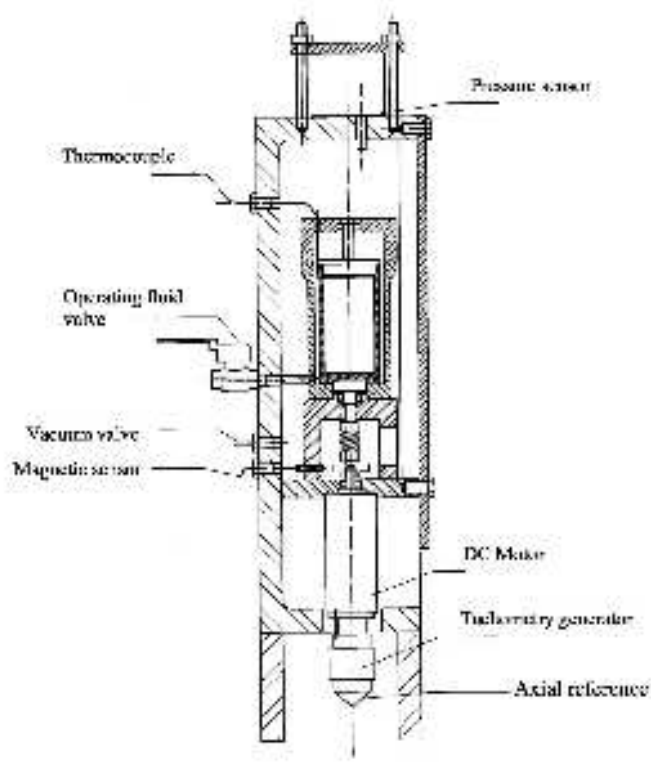

FIG. 1. General vehomatics of the apparatus.

Couette-Taylor flow, the transition from laminar to turbulent flow occurs throughout a sequence of well-defined instabilities. The focus in this study is placed on the identification of the interactions of the first three instabilities with the dispersed phase, as they occur successively in the flow. The present paper is organized as follows. In Sec. II, the experimental setup and measuring techniques are outlined. Section III focuses on visualizations of the dispersed phase and mea surements of the flow properties, with and without the two types of dispersed phase. Section IV deals with the results. In the Appendix, we describe the basic equations used to determine bubble equilibrium positions in the Couette-Taylor apparatus.

\section{EXPERIMENTAL SETUP AND MEASURING TECHNIQUES}

\section{A. Experimental setup}

The experiments were conducted in a vertical circular Couette apparatus, shown in Fig. 1, having an inner cylinder of $30 \mathrm{~mm}$ radius, $R_{i}$, an outer fixed cylinder of $35 \mathrm{~mm}$ radius $R_{o}$, and a total height $L$, of $110 \mathrm{~mm}$. The rotating angular velocity of the inner cylinder is denominated $\Omega i$. The gap, $d=R_{n}-R_{i}$, is equal to $5 \mathrm{~mm}$. The Reynolds number

$$
R_{\kappa}=\frac{2 \pi R_{i} \Omega_{j} d}{y}
$$

can be varied by changing the rotation velocity of the imner cylinder or the viscosity of the fluid, for that purpose, different glycerol-water mixtures were used. Taking into account
IABLE I Cheracteriatics of the apparatus.

\begin{tabular}{|c|c|c|}
\hline Radinn ratio & $\bar{y}=R_{1} / R_{2}$ & 0.857 \\
\hline Clearance natio & $d / R_{i}$ & 0.16 \\
\hline Boundary cenditions & $\begin{array}{l}\text { Upper } \\
\text { Lower }\end{array}$ & $\begin{array}{l}\text { Froe surface } \\
\text { Ripid fixed plate }\end{array}$ \\
\hline Ligrad beight $L$. & $\begin{array}{l}\text { Vlantiluted coufiguration } \\
\text { Crvitating coufigertion }\end{array}$ & $\begin{array}{l}100 \mathrm{~mm} \\
110 \mathrm{~mm}\end{array}$ \\
\hline Aspect natio & $\begin{array}{l}\Gamma=L / d \text { vourilatod flow } \\
\Gamma=L / d \text { cavitating flow }\end{array}$ & $\begin{array}{l}20 \\
22\end{array}$ \\
\hline Critical Roynolds mimber & $\begin{array}{l}\text { Trylor vartox flow (TVF) } \\
\text { Wavy vortax flow (WVF) } \\
\text { Modminstad wavy } \\
\text { vorter flow } \\
\text { (MWVF) }\end{array}$ & $\begin{array}{l}\mathbb{R}_{e_{1}}=102 \\
\mathbb{R}_{e_{1}}=138.4 \\
\mathbb{R}_{\theta_{2}}=1023\end{array}$ \\
\hline Nature of the fluid & $\begin{array}{l}\text { Fluid kinomatic } \\
\text { visconity } 26^{\circ} \mathrm{C}\end{array}$ & $\begin{array}{l}\text { Roypolds } \\
\text { mumber range }\end{array}$ \\
\hline $\begin{array}{l}\text { mixhure watar- } \\
\text { glycarol } 65 \%\end{array}$ & $v=113 \mathrm{ses}$ & $25<\mathbb{R} a_{a} \mathbb{R}_{a}<<0$ \\
\hline $\begin{array}{l}\text { misture watar- } \\
\text { glycarol } 70 \%\end{array}$ & $r=17 \mathrm{eS}$ & $10<\operatorname{Re}_{a} \operatorname{Re}_{01}<25$ \\
\hline $\begin{array}{l}\text { mixhure watar- } \\
\text { glycarol SON, }\end{array}$ & $v=32.57 \mathrm{es}$ & $0<R_{0} R_{e}<10$. \\
\hline
\end{tabular}

the relative uncertainty of the geometric dimensions, the viscosity estimation, and the rotational velocity described in Djéridi of al., ${ }^{7}$ the Reynolds number can be estimated to within an accuracy of $1 \%-2 \%$. In order to modify the pressure reference, the apparatus was enclosed in an airtight Plexiglas vacurum tank, which for visualization purposes was equipped with three transparent walls.

Values for the apparatus parameters and the critical Reynolds numbers corresponding to the three first instabilities are given in Table I. It is a well documented fact that the purely azinmthal flow in a circular Couette apparatus will develop a Taylor vortex flow (TVF) situation, beyond a critical Reynolds number $\mathrm{Re}_{\mathrm{cl}}=102$, where superposed counterrotating cells, with a height theoretically equal to the gap, will appear in the entire length of the apparatus. ${ }^{2}$ These cells, independent of the angular position, are known as the first Taylor instability. When the angular velocity is increased, a second instability occurs, ${ }^{9}$ corresponding to the wavy vortex flow regime and characterized by the superposition of an azimuthal wave on the initial cells, this instability is given for $\operatorname{Re}_{c 2}=138.4$. At higher Reynolds numbers, this azimuthal wave is modulated by a second wave mode, leading to a spatial flattening of the WVF, this kind of regime, called modulated wavy vortex flow (MWVF), appears at $\operatorname{Re}_{A}$ $=1023$.

The evolution of the state of the flow is known to depend largely on the way that the corresponding Reynolds number is reached ${ }^{10}$ and extreme care was talken to observe the same start up procedures for each test, thereby obtaining the best line of comparison. For all configurations, particular attention was paid to limiting the value of the acceleration of the rotational velocity (less than the threshold defined by Lim ot $a$. ") in order to ensure that the described pattems were not due to any "history effect" of acceleration. 


\section{Ventilated flow procedure}

Due to its design, the bottom of the flow domain is a fixed wall and the top boundary of the liquid is an ordinary free surface in the annulus. With an upper free surface, the natural ventilation of the flow can be obtained with a sufficiently high rotating speed of the inner cylinder. In fact, for an angular velocity larger than 13 rps (corresponding to a recuced Reynolds mumbers range $R e / R_{e l}$ between 2.5 and 4.5 ), bubbles are generated, at the crest of the overtuming breaking wave, and drawn into the liquid. In this condition, air is sucked from the free surface into the liquid. Bubbles are successively trapped by the Taylor cells along the height of the apparatus. Experiments were conducted for the Reynolds numbers range, corresponding to the first three instabilities $\left(\operatorname{Re}_{\mathrm{Re}}<20\right)$. For a large rotating velocity corresponding to the weakly turbulent flow, the free surface was excessively deformed. Therefore, it is not possible to dissociate the dispersed phase effects from the effects induced by the top boundary change.

\section{Cavitating flow procedure}

In order to observe the cavitation inception it was necessary to avoid ventilating the free surface, the liquid level in this case was greater than that set for ventilated flow ( $\Gamma$ $=22$ instead of $\Gamma=20$ ). The rotating inner cylinder was then entirely immersed. The free surface was less perturbed with no further air bubble generation. It must be pointed out that the corresponding minor increase of annulus length had no consequence on the Reynolds number for the wavy vortex flow regime transition $\mathrm{Re}_{22}$. Moreover, no significant difference was observed either in the single phase velocity profiles or in the spectrum between the ventilated and cavitating configurations.

To generate cavitation, the average reference pressure $P$. of the tank, ranging from 1000 to $10^{5} \mathrm{~Pa}$ was modified by using a vacuum pump and measured with a sensor of an absolute accuracy of $200 \mathrm{~Pa}$. Progressive decrease in the pressure was controlled by monitoring the leak rate with a valve. The accuracy of this valve allowed pressure adjustment by about $100 \mathrm{~Pa}$. To identify the cavitation inception, the mean pressure of the entire apparatus was slowly decreased at a fixed rotational velocity of the inner cylinder until cavitation appeared. In this situation, the criterion for cavitation inception can be quite different, depending on whether the appearance of the first bubbles or the appearance of organized cavitating bubbles is taken into accoumt. Detection here was based on visual observations under stroboscopic light. Considering the fact that cavitation first appeared near the bottom of the apparatus, the criterion of cavitation inception in the Couette-Taylor apparatus was determined by the observation of the first constituted bubbly ring in this region

As cavitation inception is modified by the nuclei conten of the testing fluid, ${ }^{12}$ it is of major importance to guarantee constant water quality. For that purpose distilled water was preferred to tap water and testing fluid (glycerol-water mixture) was systematically replaced between two tests. Vapor pressure of glycerol is several orders of magnitude lower than that of water. Considering that the more volatile component of a mixture is the first to evaporate when pressure is decreased, the vapor pressure of the water-glycerol mixture is taken as the value of the water vapor pressure at the operating temperature $\left(2400 \mathrm{~Pa}\right.$ at $\left.20^{\circ} \mathrm{C}\right)$.

For the simultaneous velocity measurements and flow visualizations, the water phase was seeded with microscopic particles. Taking into account the chosen criterion for cavitation inception and the small discrepancy due to the visual determination, experiments conducted with and without particles proved that the cavitation inception is not significantly affected by the presence of microscopic neutrally buoyant particles.

\section{B. Measuring techniques}

A two-component, three-beam, LDV Dantec system was used to measure azimuthal and axial velocities. The system was operated in backscattering mode and was coupled with two Dantec enhanced burst spectrum analyzers. The largest dimension of the measuring volume (40 $\mu \mathrm{m} \times 40 \mu \mathrm{m}$ $\times 500 \mu \mathrm{m})$ corresponded to one tenth of the gap. Extreme care was taken to determine the position of the measurement volume within the gap, taking account of the deviation of the beams through the outside cylinder. The deviation was due to the refractive index variation and the particular geometry of the interface. The velocities were measured according to a refined grid in the gap. The isovelocity contours presented in the following sections were obtained over a measurement grid of 651 points with a $0.25 \mathrm{~mm}$ spacing in the radial direction and a $0.5 \mathrm{~mm}$ spacing in the axial direction (corresponding, respectively, to $0.05 d$ and $0.1 d$ ). In order to quantify the impact of the azimuthal instability, a spectral analysis was performed on a time series of signals in the gap for different Reynolds numbers. The data rate, $3 \mathrm{kHz}$, was sufficiently high, for the above LDV measurements, compared to the characteristic frequencies of the flow. The random signal was resampled at equidistant intervals of time, using a sample-and-hold interpolation method, to preserve the amplitude information, before applying a fast Fourier transform algorithm.

\section{RESULTS}

We examine the gas/vapor phase arrangement in ventilated and cavitating flows. The effects of the gas/vapor phase on the structures of the Couette-Taylor flow are shown through a quantitative investigation of the liquid velocity field Results from ventilated and cavitating flows are deliberately combined, in order to highlight common points and differences.

\section{A. Gas phase arrangement \\ 1. Ventilated flow}

Figure 2 shows the particular gas phase pattem for different Reynolds numbers. $R e / \operatorname{Re}_{c 1}=3.5$ and 4.5 [Figs. 2(a) and 2(b)], referring to Djéridi of $a l^{7}$ The bubbles produced by the agitation of the free surface are captured by the organized structures of the flow (Taylor cells) and are arranged 


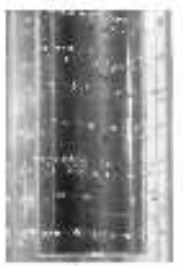

is:

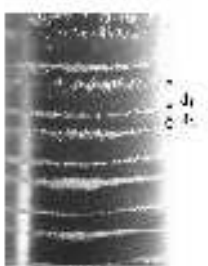

is

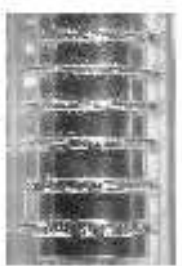

FIG. 2 Vimalieations of the organised gaseous phase in the gap for difforent reduced Reypolds mmeber (vewalstod flow toufiguration). String of bebbles ahtemate vertically with distances: $d_{1}$ and $d_{2}$. (a) $\mathrm{Re} \mathrm{Re}_{\mathrm{c}}$ $=3.5$, (b) $\mathbb{R} e / R_{a r}=4.5$, and $(c) \mathbb{R}_{0} \mathbb{R}_{a_{\mathrm{c}}}=11$

along the apparent azimuthal waves, like strings. These strings altemate vertically at distances of $0.8 d$ and $1.2 d$. Along each wave, individual spherical bubbles are arranged as if queuing between elongated clouds of aggiomerated individual spherical bubbles. The largest bubbles are at the top and the smallest at the bottom. In Fig. 2(c), for $R e / R e$ il $=11$, the aspect of bubble rings is different. Here, we observe the formation of a gas filament localized near the immer wall of the apparatus in the outflow regions. This kind of configuration was observed in situations beyond reduced Reynolds numbers $\operatorname{Re} \mathrm{Re}_{e l}$ between 7.2 and 7.5 .

\section{Cavitating flow}

The vapor phase arrangements are shown in Figs. 3(a)3(c) for different reduced Reynolds numbers and for an ambient pressure of around $2500 \mathrm{~Pa}$. When the pressure is progressively decreased, for a fixed rotational velocity corresponding to the TVF regime, only upward-traveling bubbles appear, governed by the broyancy force, and no developed cavitating regime can be attained On the other hand, for the WVF regime and a sufficient rotational velocity, the pressure drop leads to the appearance and capture of bubbles. Instead of a filled vapor tube as can be observed in other kinds of cavitating vortices, the vapor phase is organized like strings of individual bubbles. As observed in the ventilated flow, bubbles are aligned along the azimuthal waves, in the

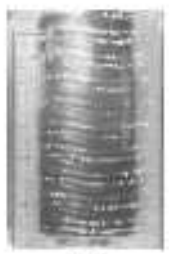

(a)

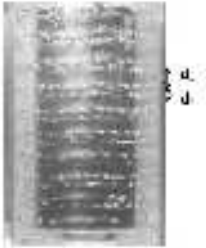

(b)

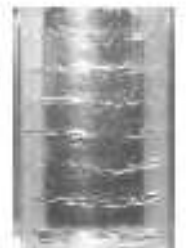

scl
FIG. 3. Vhunlizations of the organized vapor phase in the gap for differrat reducad Regnolds mmbers (cantating flow cosfigmation for P. $=2500 \mathrm{P}_{2}$ ) - Serings of bebblen alinamate trertically with distances $d_{3}$

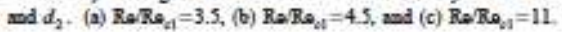

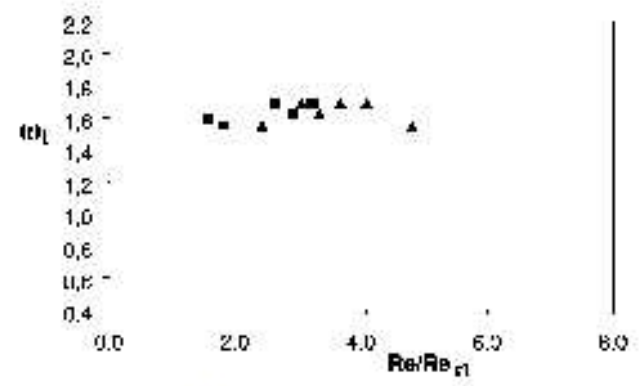

FIG. 4. Evolution of the axinuthal wsse dimemionless freqpency with ro daced Roynolds mambar for single phase and voutilated flown: Iingle phase flow and $\boldsymbol{A}$ wantilated flow:

core of the vortex structures, with alternating distances $d_{1}$ and $d_{2}$, respectively, equal to $1.2 d$ and $0.8 d$ for $R_{e} R_{e l}$ $=3.5$.

These distances change progressively as the Reyuolds number increases, $d_{1}$ becoming larger and $d_{2}$ smaller, up to a point where the bubble rings fuse in the outflow regions near the inner cylinder beyond $\mathrm{Re}_{\mathrm{Re}}$, which is set between 7.2 and 7.5. At this final state, the bubbles have an elongated shape. They are more dispersed along the azimuthal waves, constituting larger rings. This configuration can be observed in Fig. 3(c) where $\mathrm{Re}_{\mathrm{Re}}=11$ as in the ventilated flow regime.

\section{B. Effects on the flow structure}

To provide an accurate velocity measurement of the liqvid phase with classical LDV, the measurements in radial and axial directions were performed at $R e / R e_{s 1}=10$. Indeed, the localization of a vapor'gas filament near the inner wall of the apparatus, in cavitating or ventilated configuration enables liquid velocity measurements elsewhere in the gap. For the lower Reynolds number range, when bubbles are arranged along the azimuthal wave like strings near the core of the Taylor cells, in cavitating or ventilated flow, the LDV signal can be affected by the signature of the bubbles. In these conditions, only a spectral analysis was performed on a se ries of signals in the flow region where no strings of bubbles were evident.

\section{Ventilated flow}

Measurements were performed in the WVF regime. In single flow, it is well known that this regime is characterized by an azimuthal wave of findamental frequency $\omega_{1}$ (normalized by the velocity of the inner cylinder $\Omega_{i}$ ) equal to 1.66. In Fig. 4 this frequency is reported as a function of $\mathrm{Re}_{\mathrm{Re}}$ ranging between 2.5 and 5 and for single and ventilated flows (conesponding to an arrangement of string-like bubbles located in the Taylor cells). This nondimensional frequency remains clearly ummodified by the presence of the gas phase. In order to clarify a possible modification of the flow structure, a more detailed investigation was conducted for $\operatorname{Re}_{\mathrm{Re}} \mathrm{el}=3.5$. Under these conditions, Fig. 5 presents the spectrum of the axial velocity performed in single phase flow 


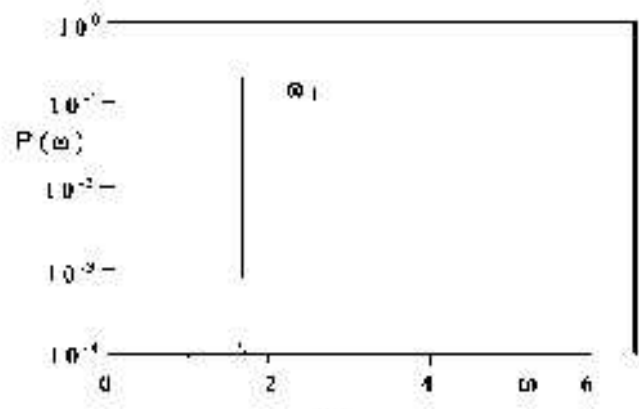

(a)

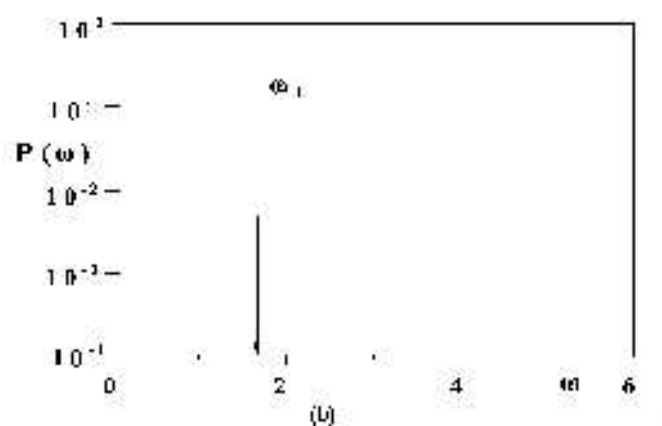

FIG. 5. Comparivon of spectra obtained at $\mathbb{R e} \mathbb{R} e_{4}=3.5$ : (a) single-phase Blox and (b) veotilatad flox.

[Fig. 5(3)] and in ventilated flow [Fig. 5(b)]. On both spectra, only one fumdamental ray of frequency $\omega_{1}$ was observed. indicating that the gaseous phase has no effect on the flow structure for low Reynolds numbers in the WVF regime.
Let us consider now the case where bubbles are situated in the outflow regions near the imer cylinder. Figures $6(a)$ and $6(\mathrm{~b})$ present isocontours of axial velocity in both the ventilated and the single phase WVF regimes at $R e R_{e}$ $=10$. The evolution of nondimensional axial velocity, normalized by $y / d$ following a linear increment, is displayed in the $(x, z)$ plane for a nondimensional radial position $x$ $=r / d-\left(R_{i}+R_{b}\right) / 2 d$. Figures $6(\mathrm{a})$ and $6(\mathrm{~b})$ give an example of the positive and negative components illustrating the well-known Taylor vortex structure. At $\operatorname{Re}_{\mathrm{Re}}=10$, no noticeable influence of the gaseous phase is evident on the nondimensional value of the axial velocity. In the single phase flow, the axial wavelength $\lambda$, equal to $2 d$, is defined as the axial length of a pair of coumter rotating vortices (equivalent to $2 L / n$, where $L$ is the height of the fluid column and $n$ is the number of Taylor vortices). But, at the same Reynolds number in the ventilated flow [Fig. $6(\mathrm{~b})$ ], the axial wavelength is found to be much larger than that of the single phase flow (extended some $45 \%$ with the value of 2.9 d).

As a result, the structure of the flow is modified by ventilation somewhere in the interplay for $\mathrm{Re} / \mathrm{Re}_{\mathrm{cl}}$ between 5 and 10. Spectral analysis was then performed on the axial velocity component at $R e / R_{e l}=7.5$. For this configuration the dispersed phase was situated in the outflow regions near the inner cylinder. The spectra obtained for single phase and ventilated flows in Figs. 7(a) and 7(b) show that the normalized azimuthal wave frequency $\omega_{1}$ characterizing the WVF regime is rechuced to 1 by the gas phase. The main point is that no other fundamental frequency is introduced in the twophase WVF. Nevertheless, complementary stroboscopic measurements show that the associated wave unumber $m_{3}$, shifts from 5 to 3 . Therefore, the phase velocity defined as $\omega_{1} / m_{1}$ remains the same in single phase and ventilated flows and is equal to 0.33 . As a conclusion for large Reynolds numbers in the WVF, the introduction of the ventilated phase leads to
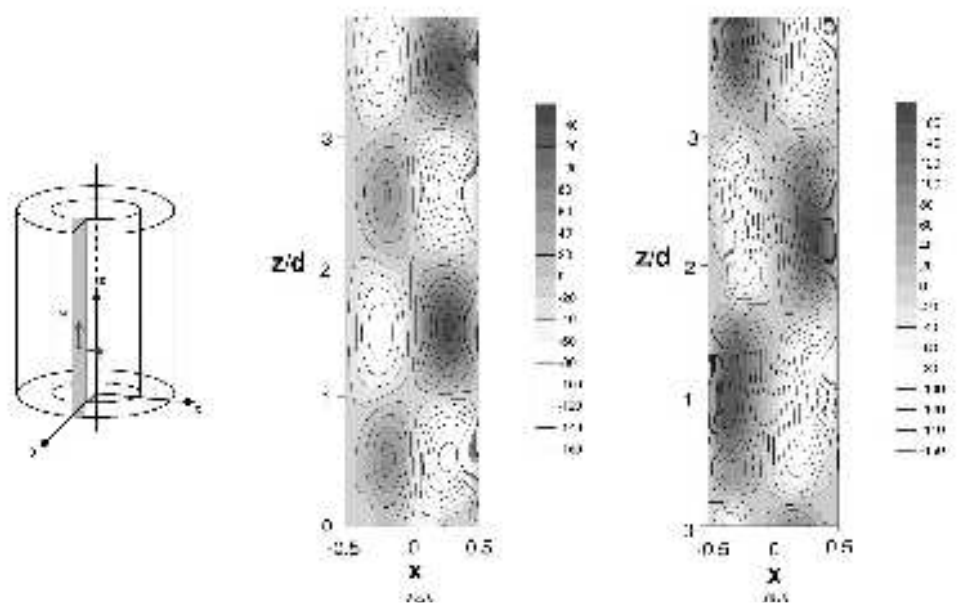

FIG. 6. Comprision of the ivocontourn of dimescrionlens axil trolocity com ponont at $\mathbb{R} e \mathbb{R}_{e_{1}}=10$ : (a) siagle phase flow and (b) reetilated flow. 


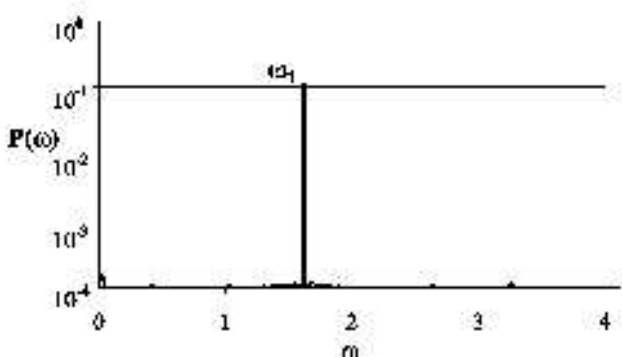

(n)

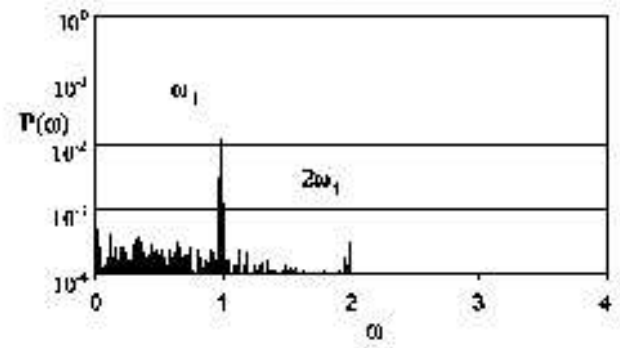

(b)

FIG. 7. Comparison of spectra obtained at $\mathbb{R} e / \mathbb{R}_{a 1}=7.5$ : (a) ingle-phase flow and (b) ventilated florx.

a change in both the axial wavelength and the azimuthal wave number.

\section{Cavitating flow}

The evolution of the normalized azimuthal wave fre quency $\omega_{1}$ and azimuthal wave number $m_{1}$ were measured under stroboscopic lighting and are presented in Fig. 8 according to the Reynolds number for the WVF and MWVF for both single-phase flow and cavitation conditions. In the single-phase flow, two discontinuities of the azimuthal wave frequency are observed for reduced Reypolds numbers of 10.5 and 16. These discontinuities have already been described by Fenstermacher of $a l^{13}$ The previous authors focused on the fact that these frequency shifts comespond to a modification of the wave number as shown in Fig. 8. For cavitation conditions, the modifications of the frequency and wave number occur earlier for reduced Reynolds numbers equal to 7.2 and 13 . Note that cavitation, obtained either by decreasing the pressure or by increasing the inner cylinder rotational velocity yields the same results. As in the ventilated regime, for a low Reynolds number range 3.5 $<\operatorname{Re} \operatorname{Re}_{\mathrm{cl}}<7$, the azimuthal frequency remains unchanged in the presence of bubbles and equal to 1.66. Cavitation has no significant influence on the phase velocity $\omega_{1} / m_{1}$ up to a reduced Reynolds number of 7 , and equals 0.33 (value observed by Coles $^{14}$ and Shaw et $a l^{15}$ in single-phase flow).

Spectra are presented in Figs. 7(a) and 9, respectively, for single-phase flow at $\operatorname{Re}_{\mathrm{Re}}=7.5$ and for cavitating flow at $\operatorname{Re}_{R_{e}}=7.2(\mathrm{Pe}=3000 \mathrm{~Pa})$. For the cavitating flow, a

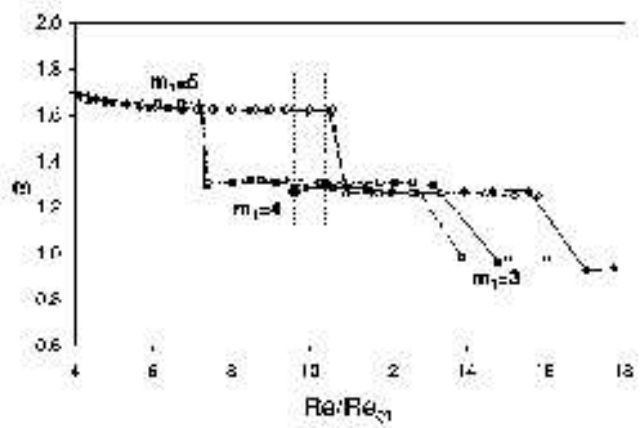

FIG. 8. Evolution of the arimuthal wave dimonsionlon froquency and the wave mumber va Roypolds nmmber (ropestability is imsortigatod solid and

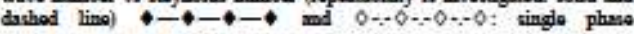

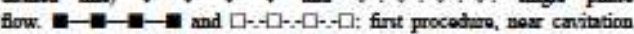
incoption, for a fixed vacumm tank prossure $\left(\mathrm{Po}_{2} \sim 2700 \mathrm{P} 2\right)$, by decreasing

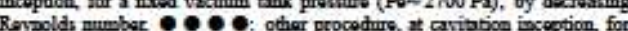
different fixed Reypolds mmbers, by decreasing Po.

new incommensurable frequency emerges. This observation can be interpreted as an early indication of the fhird instability.

The presence of the third instability can also be visualized using a stroboscope to freeze the azimuthal wave, the onset of the MWVF being characterized by the periodic flattening of the vortex cutflow boundary. In the cavitating regime, this phenomenon was observed beyond a reduced Reynolds between 7.2 and 7.5 instead of the usual critical value of 10.5 .

In Figs. 6(a) and 10, isocontours of axial velocity are plotted at $\operatorname{Re} R \mathrm{Re}_{\text {I }}=10$ for the single-phase flow and the cavitating flow ( $\mathrm{Pe}=2500 \mathrm{~Pa}$ ). Measurements show clearly that the axial wavelength is increased by $25 \%$ with the vapor phase ( $2.5 d$ rather than $2 d$ in the single-phase flow).

In conclusion, cavitation leads to an early appearance of the third instability, a premature change in the second instability's azimuthal wave number and an increase in the axial wave length.

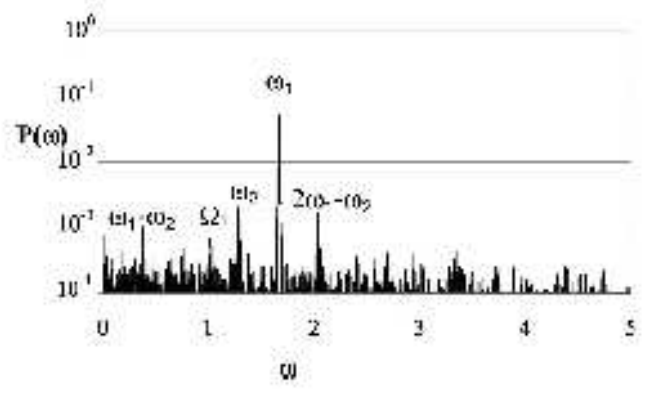

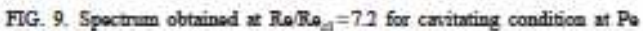
$=3000 \mathrm{~Pa}$. 


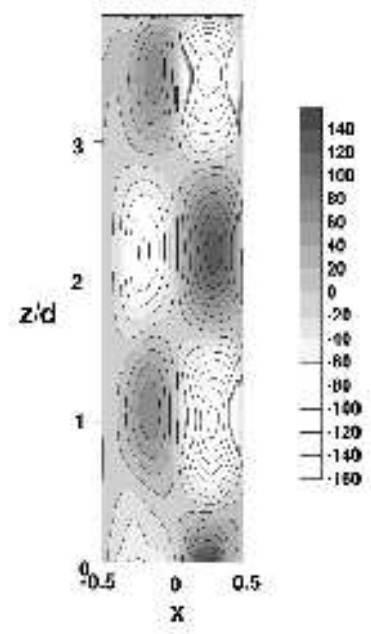

FIG. 10. Inocoutours of the axial vilocity componant at $\mathbb{R e} / \mathbb{R}_{4}=10$ for cavitating condition at $\mathrm{Pe}=2500 \mathrm{~Pa}$

\section{DISCUSSION}

A. Behavior of the dispersed phase: Passive or active tracer?

To answer the above question, we need to take a close look at similarities and differences observed in the flow according to the Reynolds number both for single- and twophase flows.

For $\mathrm{Re}_{\mathrm{Re}}<1<7.2-7.5$, both for ventilated and cavitating flows, the visualizations show that the bubble strings are located in the core of the Taylor cells and that the corresponding axial wave length is not altered by the dispersed phase Moreover, the presence of the bubbles does not lead either to the introduction of another fimdamental frequency or to the modification of the azimuthal wave number. For this Reynolds numbers range, the dispersed phase can be considered as a passive tracer

On the contrary, for $\operatorname{Re}_{\mathrm{Re}_{\mathrm{e}}}>7.2-7.5$, the visualizations show that the dispersed phase migrates to the outfiow regions. In the ventilated regime, the axial wave length is in creased and the azimuthal wave frequency is decreased but the transition from WVF to MWVF, characterized by the appearance of a second fundamental frequency, does not appear earlier than in single-phase flow. In the cavitating regime, the same tendencies are observed conceming the axial wave length and the azinuthal wave frequency but the transition from WVF to MWVF appears earlier. We can then conclude that, for $\operatorname{Re} \operatorname{Re}_{1} \geqslant 7.2-7.5$, the dispersed phase is an active tracer both for ventilated and cavitating flows.

\section{B. Analysis of the dispersed phase arrangement}

In order to understand the bubble capture in the Taylor cells, it is necessary to analyze the force budget applied to a bubble. For a low Reynolds number range in the WVF IEgime $\left(4.5<\operatorname{Re}_{\mathrm{Re}}<7.2\right)$, the dispersed phase is considered as a passive tracer. Thns, the bubble equilibrium positions can be determined analytically in the undisturbed flow. The axial and radial velocities from the Davey's expansion theory ${ }^{16}$ (extended to larger Reynolds numbers according to more recent works ${ }^{17}$, as well as an experimental radial profile of azimuthal velocity, were implemented to calculate the bubbles' equilibrium positions. We considered the azinuthal wave as if it were frozen and the flow as if it were steady and $\theta$ independent.

The equation that governs the bubble motion is given by the balance of forces acting on the bubble:

$$
\begin{aligned}
& -\frac{\pi r_{b}^{2}}{2} \rho_{j} C_{D} \mid \mathbf{V}_{\mathrm{b}}-\mathbf{V}_{\mathrm{d}}\left(\mathbf{V}_{\mathrm{b}}-\mathbf{V}_{\mathrm{t}}\right)+\left(\frac{4}{3} \pi r_{b}^{3}\right) \rho_{f} \\
& \times\left[\frac{D \mathbf{V}_{f}}{D t}+C_{a}\left(\frac{D \mathbf{V}_{f}}{D t}-\frac{d \mathbf{V}_{b}}{d t}\right)-C_{l}\left(\mathbf{V}_{\mathrm{b}}-\mathbf{V}_{f}\right) \wedge \Omega_{r}-\mathbf{g}\right]=0,
\end{aligned}
$$

where the different terms on the left-hand side represent drag, pressure gradient, added mas5, lift, and buoyancy forces, respectively, all the while ignoring the bubble inertia. In this simple model, interactions between the bubbles are ignored and bubbles are considered as isolated spherical bubbles of radius $r_{b}$. $\rho_{f}$ is the liquid density, $g$ the acceleration of gravity, $\mathbf{V}_{\mathbf{b}}$ the bubble velocity, and $\mathbf{V}_{\mathrm{f}}$ the liquid velocity at the center of the bubble. The different forces are expressed in the cylindrical coordinates system in the Appendix.

In the present study, the Reynolds number $\mathrm{Re}_{3}$ of the bubble is lower than 10 , and the expression of the drag coefficient has been defined by Magnandet of $a l:{ }^{18}$

$$
C_{D}=\frac{16}{\operatorname{Re}_{b}}\left(1+0.16 \operatorname{Re}_{b}^{0.5}\right) \text { for } \operatorname{Re}_{b}=\frac{\mid \mathbf{V}_{\mathbf{b}}-\mathbf{V}_{\mathrm{r}} \mathrm{d}\left(2 r_{\mathrm{b}}\right)}{y} \text {. }
$$

The added mass coefficient $C_{a}$ is taken as equal to its standard value of 0.5 given by Auton. ${ }^{19}$

Concerning the lift force, we faced three parameters of influence: the bubble Reynolds number, the vorticity, and the strain. In our case, the bubble Reynolds number is very low, the vorticity is high, and the strain is not to be discounted. In those conditions, the review of the literature shows that developed models ${ }^{19}, 20$ were not entirely suitable to our requirements. We, therefore, opted for Auton's formulation with a constant coefficient and we tested a large range $(0.5-3)$ of lift coefficients, which led to equilibrium positions situated close to each other (maximsl relative difference in the $z$-equilibrium position of $10 \%$ ).

To compute the bubble equilibrium positions, the velocity and acceleration of the bubble in the $(r, z)$ plane were set at zero $\left(u_{b}=0, w_{b}=0, d u_{b} / d t=0 d w_{b} / d t=0\right)$. If we consider that the bubble velocity in the azimuthal direction is steady $\left(d v_{b} / d t=0\right)$, the azimuthal drift velocity is deduced from the azimuthal balance of the forces. This drift velocity component is expected to be small and results in 


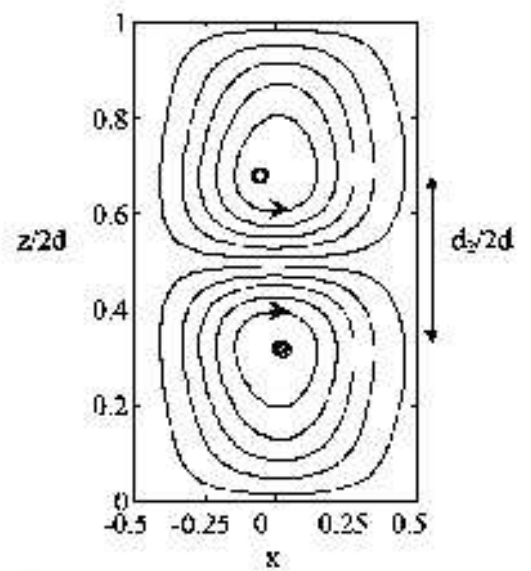

FIG. 11. Recults of force budget Equilibrimm pocition of a $1 \mathrm{~mm}$ bubble in the gap at $\mathbb{R} e / \mathbb{R}_{e_{41}}=4.8\left(C_{1}=0.5\right)$.

$$
v_{b}-v_{f}=\left(1+C_{s}-C_{l}\right) \frac{r_{b}^{2}}{3 v} \frac{u_{f}}{\left(1+0.16 \mathrm{Re}_{3}^{0.5}\right)}\left(\frac{v_{f}}{r}+\frac{\partial v_{f}}{\partial r}\right) .
$$

By injecting this azimuthal drift velocity in the radial and axial force budget, bubble equilibrium positions were determined. The linear dynamical system of the motion of the bubbles near their equilibrium position was then expressed as a matrix product. The determination of the Jacobian eigenvalnes, which have negative real parts, led to the determinstion of the stable equilibrium positions. This system is described in detail in the Appendix.

Figure 11 represents the computed equilibrium positions in the gap for a $1 \mathrm{~mm}$ bubble, a reduced Reynolds number $\mathrm{Re} / \mathrm{Re}_{\mathrm{cl}}=4.8$, and a mixture of $20 \%$ water and $80 \%$ glycerol. The distance between two consecutive bubble rings is alternately $d_{1}$ and $d_{2}$ as previously quoted in the description of the experiments. As expected, these positions are located near the core of the cells on both sides of the outflow region. The estimated aximuthal difif velocity is smaller than $1 \%$ of the liquid azimuthal velocity, leading to a small Weber number.

$$
\text { We }=\frac{\rho d v_{b}-v d^{2} r_{b}}{s},
$$

$s$ being the surface tension of the gas/contimnous, vapor continuous phase. The maximum value of We is of the order of $0.1 \%$, corresponding to the observed spherical shape of the trapped bubbles.

These theoretical alternating distances were calculated for different Reynolds numbers, and then compared to the experimental results observed using a mixhure of $20 \%$ water and $80 \%$ glycerol. These alternating distances are given in Fig. 12(a). We notice that the computed results do confinm the observed distances between the bubble rings. Nevertheless, it is of note that the computed equilibrium positions are
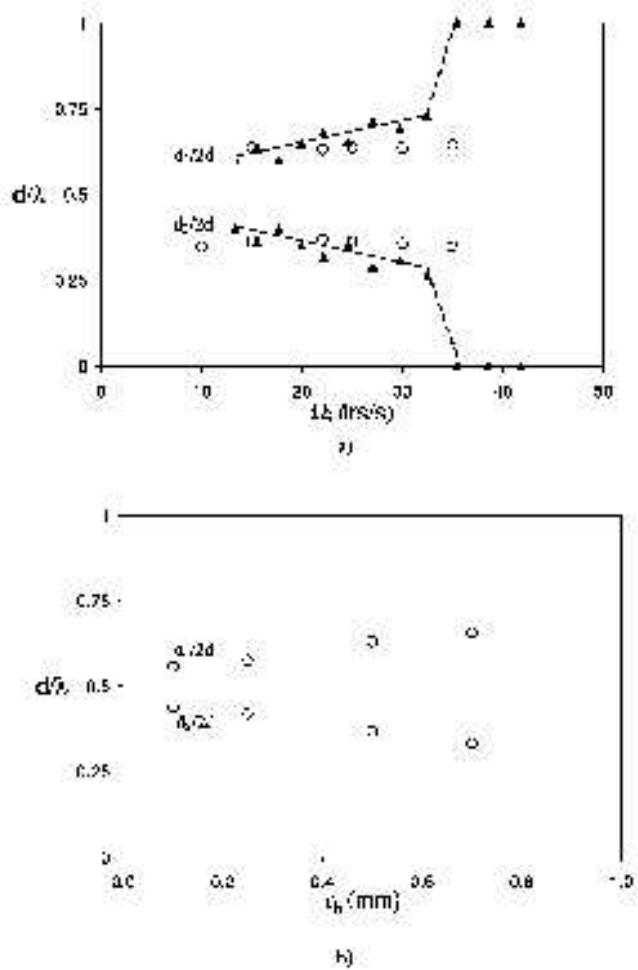

FIG. 12. Evobrtion of the distances betwven two ceasecutive bubble

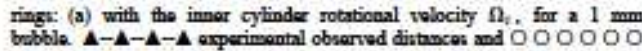

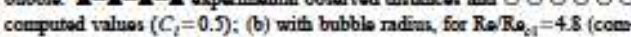
puted vahos with $C_{l}=0.5$ ).

not as sensitive to the rotational velocity of the inner cylinder as was observed in the course of this experiment. This can be mainly explained by two reasons related to the estimation of drag and lift coefficients. First, had we taken into accoumt the vorticity in the expression of the drag coefficient, ${ }^{21}$ the drag force would have increased. In fact, the axial component of the drag force brought bubbles nearer the outflow region. Second, at the equilibrium positions near the core of the Taylor cells, the main contribution to strain is the azinuthal flow. Had we taken into account this significant contribution in the formmlation of the lift force, both the azimuthal drift velocity and the radial equilibrium positions, as well as the $z$-equilibrium positions would have been quantified more precisely.

The previous calculations were performed for a $1 \mathrm{~mm}$ bubble, but it must be pointed out that the dispersed phase was constituted of different bubble sizes. For $\operatorname{Re} \mathrm{Re}_{\mathrm{el}}=4.5$ using image processing, a bubble size range was determined between 0.1 and $1.5 \mathrm{~mm}$. Figure 12(b) illustrates these computed altemative distances in relation to the bubble size. The bubble size increase leads to a displacement of the computed 
stable equilibrium positions to nearer the outflow regions Therefore, if a critical bubble size is surpassed, equilibrium positions no longer exist in the core of the cells. From the experimental results, this modification of the equilibrium positions corresponds to an observed migration of the bubbles towards the inner cylinder in the outflow regions. The computation revealed that critical size decreases when rotational velocity increases.

Due to the active behavior of the dispersed phase for the larger Reynolds number range corresponding to migration, no calculation of the bubble trajectory or location is available but we shall now focus on the phenomenology of the migration process.

For those Reynolds numbers, the flattening of the azimuthal velocity profile induces an increase in the radial gradient of azimuthal velocity near both cylinders. Those higher velocity gradients will be at the origin of an increase in both the azimuthal drift velocity [see Eq. (3)] and the radial lif force (see the Appendix). This force is directed towards the inner cylinder and will induce displacement of the bubbles' equilibrium positions. Finally, no stable equilibrium positions are found near the core of the Taylor cells and bubbles migrate to the inner cylinder in the outflow region. The increase in the azimuthal drift velocity is thought to be responsible for the bubble deformation, as experimentally observed in this region of the flow.

\section{Analysis of the interaction mechanism}

The gaseous or vapor phase gives rise to flow modifications when the dispersed phase migrates to the outflow region in the regimes of the second and third instabilities. In this case, interaction between the liquid phase and dispersed phase is characterized by an increase in vortex axial length and a change in the state of the flow for the WVF regime

In the present experiment, the introduction of the dispersed phase is likely to induce a modification of both average density and viscosity, this modification being effective in the zone where the dispersed phase is located. Thus the migration of the dispersed phase into the outflow region near the inner cylinder leads to a radial discontinuity of both density and viscosity. When conditions were such, instabilities were boosted. Renardy and Joseph ${ }^{22}$ numerically highlighted that the Couette-Taylor flow is more stable with a thin layer of less viscous or lighter fluid next to the inner cylinder than with the less viscous or lighter fluid next to the outer cylinder As the distribution of density and viscosity are similar, even though laws of evolution of both quantities are not the same, some other mechanism must be involved to explain the interactions.

These interactions between the liquid phase and the dispersed phase are partly characterized by a distortion of the Taylor cells illustrated by an increase in the axial wave length, in both ventilated and cavitating flows. The same trend was observed by Gopalan and Katz. ${ }^{23}$ They show that, under certain conditions, even with few entrained bubbles at very low void fractions, the core of laminar, transitional, and turbulent vortices is elongated and distorted. Similar behavior is obtained in the numerical simmlation of the mixing layer, ${ }^{4}$ by highlighting the role of the residence time of bubbles in the vortex core and the influence of the bubbles accummlation in the vortex.

Taking into account that the size of the Taylor cells was not modified when bubbles are trapped inside the cells, the residence time or accumulation effects can be discounted. In fact, the elongation of Taylor cells appears when the bubbles are located in the outflow region near the immer cylinder. It can be deduced that the bubbles migration process induces a change in the liquid radial velocity and thns a change in the size of the cells. This leads us to believe that momentum exchange between the phases explains the observed flow modifications. In the ventilated flow, the increase in the Taylor cells size can also be explained by the axial flux induced by the bubble migration from the top to the bottom of the apparatus, as is observed in a single phase Couette-Taylor flow when an axial flux is superimposed.

Interactions between the liquid phase and the dispersed phase are also characterized by an earlier appearance of the third instability, particularly for the cavitaring flow. Let as now discuss the reason for this earlier transition.

Compared with the ventilated regime, early evidence of the third instability is most likely associated with the specific presence of the vapor phase. Thus, the cavitation phenomenon may infroduce another type of instability superimposed on those existing in the single phase flow. However, vapor interface instabilities are characterized by much higher frequencies than those found in the flow. Therefore, this type of instability must be discounted as an explanation of the earlier transition to the third instability, given the scales implied.

In single phase flow, the transition to the third instability is accompanied by a steepening of the velocity gradients at the inner and outer cylinder. It corresponds to an increase in radial transport of fluid azimuthal momentum from near the inner wall outward to outflow regions between vortices. ${ }^{24}$ Therefore, the transition to the third instability in a singlephase flow is undoubtedly linked to an increase in the local wall shear stress. There is no reason why the same should not be in the Conette-Taylor two-phase flow when bubbles are located near the inner cylinder. In a two-phase flow, when bubbles are located in the near wall regions (in a vertical upward pipe flow ${ }^{25}$ or in a borizontal boundary layer with bubble injection at the wall ${ }^{26}$ ), wall shear stress is known to be greater than that obtained in the single phase flow. This behavior is linked to bubble drift velocity and especially to the void fraction concentration near the wall

Taking into accoumt that bubble size and dynamics are similar in the cavitating and ventilated Cowette-Taylor flows, the advanced transition to the third instability observed in the cavitating flow can be explained by a higher void fraction. Due to the small dimension of the apparatus, no intrusive measurements of the local void fraction were possible However, image processing based on a segmentation method ${ }^{27}$ gave a quantitative idea of the global void fraction in the gap. At $R e \operatorname{Re}_{s 1}=7.5$, the global void fraction is $12 \%$ and $17 \%$, respectively, for the ventilated and cavitating flows (at cavitation inception). When the void fraction increases, wall shear stress is expected to be more significant leading to earlier transition to a higher order instabilitv. 
Further work is currently in progress concerning the local determination of void fraction and bubble drift velocities with fiber-optic probes and the determination of wall shear stress with a hot film probe in a larger Couette-Taylor apparatus.

\section{v. CONCLUSION}

This study provides the evidence of mutual interactions between the instabilities of the Conette-Tayior flow and a noncondensable (ventilated flow) and condensable (cavitating flow) dispersed phase.

For a low Reynolds numbers range $(2.5<\operatorname{Re} / \operatorname{Re}$ $<7.2-7.5$ ), the dispersed phase takes the form of bubble rings agglomerated in the vicinity of the Taylor cells core The dispersed phase behaves as a passive tracer. The axial wave length and aminuthal wave number are not modified and there is no introduction of any other findamental frequency.

For larger Reynolds numbers $\left(7.2-7.5<\operatorname{Re} / \operatorname{Re}_{e 1}<20\right)$, a particular arrangement of the dispersed phase close to the imer cylinder wall in the ourflow regions is responsible for modifications in the primary instabilities. The introduction of the noucondensable phase leads to growth in the axial wavelength and change in the second instability's azimuthal wave unmber. The same trends are observed with the introduction of a condensable phase but this time early appearance of the third instability is established.

Differences observed between the noncondensable and condensable phase effects on the flow are attributed to the void fraction. Indeed, in this specific study, the void fraction is more significant in the cavitating flow than in the ventilated flow. Therefore, the void fraction and the ensuing momentum exchange between the liquid and dispersed phase explain the observed modifications in the liquid flow.

In conclusion, these experimental results can be interpreted as an early indication of turbulence development due to the introduction of a dispersed (noncondensable or condensable) phase.

\section{ACKNOWLEDGMENTS}

This work was subsidized by the French Navy. The antthors are grateful to the crew of the Mechanical Department workshop for their technical assistance. The authors are also gratefil to C. Griffin for her partial proof reading.

\section{APPENDIX: BUBBLES' EQULLIBRIUM POSITIONS IN} THE TAYLOR CELLS

The forces applied on a spherical bubble are introduced in Sec. IVB. The dynamical system is written in the cylindrical coordinate system $(r, \theta, z)$.

When bubbles are trapped by the Taylor cells, their drift velocity is reduced to its azimuthal component $v_{b}-v_{f}$. Bubbles behave like passive tracers with respect to the flow, and their drift velocity is small. In this context, the drag coefficient $C_{d}$ is equal to $16 / \mathrm{Re}_{b}$, allowing us to linearize the drag force as

$$
\mathbf{F}_{d}=-4 v \pi r_{b} \rho_{f}\left\{\begin{array}{c}
u_{b}-u_{f} \\
v_{b}-v_{f} \\
w_{b}-w_{f}
\end{array}\right\} .
$$

The pressure gradient force components are

$$
\mathbf{F}_{\mathrm{p}}=\frac{4}{\tau} \pi r_{h}^{3} \rho_{f}\left\{\begin{array}{c}
-\frac{v_{f}^{2}}{r}+u_{f} \frac{\partial u_{f}}{\partial r}+\frac{v_{f}}{r} \frac{\partial u_{f}}{\partial \theta}+w_{f} \frac{\partial u_{f}}{\partial z}+\frac{\partial u_{f}}{\partial t} \\
\frac{u_{f} v_{f}}{r}+u_{f} \frac{\partial v_{f}}{\partial r}+\frac{v_{f}}{r} \frac{\partial v_{f}}{\partial \theta}+w_{f} \frac{\partial v_{f}}{\partial z}+\frac{\partial v_{f}}{\partial t} \\
u_{f} \frac{\partial w_{f}}{\partial r}+\frac{v_{f}}{r} \frac{\partial w_{f}}{\partial \theta}+w_{f} \frac{\partial w_{f}}{\partial z}+\frac{\partial w_{f}}{\partial t}
\end{array}\right\} .
$$

For the added mass force, we obtain

$$
\mathbf{F}_{a}=C_{a} \frac{4}{1} \pi r_{s}^{3} \rho_{f}\left\{\begin{array}{c}
\frac{\left(v_{b}^{2}-v_{f}^{2}\right)}{r}+u_{f} \frac{\partial u_{f}}{\partial r}+\frac{v_{f}}{r} \frac{\partial u_{f}}{\partial \theta}+w_{f} \frac{\partial u_{f}}{\partial z}+\frac{\partial u_{f}}{\partial t}-\frac{d u_{b}}{d t} \\
r \\
\frac{\left(u_{f} v_{f}-u_{b} v_{b}\right)}{r}+u_{f} \frac{\partial v_{f}}{\partial r}+\frac{v_{f}}{r} \frac{\partial v_{f}}{\partial \theta}+w_{f} \frac{\partial v_{f}}{\partial z}+\frac{\partial v_{f}}{\partial t}-\frac{d v_{b}}{d t} \\
u_{f} \frac{\partial w_{f}}{\partial r}+\frac{v_{f}}{r} \frac{\partial w_{f}}{\partial \theta}+w_{f} \frac{\partial w_{f}}{\partial z}+\frac{\partial w_{f}}{\partial t}-\frac{d w_{b}}{d t}
\end{array}\right\} .
$$

The resulting lift force is

$$
\mathbf{F}_{1}=C_{1} \frac{4}{3} \pi r_{b}^{3} \rho_{f}\left\{\begin{array}{l}
\left(w_{b}-w_{f}\right)\left(\frac{\partial u_{f}}{\partial z}-\frac{\partial w_{f}}{\partial r}\right)-\left(v_{b}-v_{f}\right)\left(\frac{\partial v_{f}}{\partial r}+\frac{v_{f}}{r}-\frac{1}{r} \frac{\partial u_{f}}{\partial \theta}\right) \\
\left(w_{b}-w_{f}\right)\left(\frac{\partial v_{f}}{\partial z}-\frac{1}{r} \frac{\partial w_{f}}{\partial \theta}\right)+\left(u_{b}-u_{f}\right)\left(\frac{\partial v_{f}}{\partial r}+\frac{v_{f}}{r}-\frac{1}{r} \frac{\partial u_{f}}{\partial \theta}\right) \\
-\left(v_{b}-v_{f}\right)\left(\frac{\partial v_{f}}{s z}-\frac{1}{-} \frac{\partial w_{f}}{\Delta \theta}\right)-\left(u_{b}-u_{f}\right)\left(\frac{\partial u_{f}}{s-}-\frac{\partial w_{f}}{s-}\right)
\end{array}\right\} .
$$


To compute the passive bubble location in the Taylor cells, the azimuthal wave is frozen considering the flow to be steady and $\theta$ independent. Under these assumptions, the bubble equilibrium requires that the bubble stand motionless in the $(r, z)$ plane and that the azimuthal drift velocity satisfies the equilibrium of the forces in the azimuthal direction. Taking into account the axially averaged azimuthal velocity profile $v_{f}$, it yields

$$
\begin{aligned}
& u_{b}=w_{b}=0, \quad \frac{d u_{b}}{d t}=\frac{d v_{b}}{d t}=\frac{d w_{b}}{d t}=0, \\
& v_{b}=v_{f}+\frac{r_{b}^{2}\left(1+C_{a}-C_{l}\right)}{3 v} u_{f}\left(\frac{v_{f}}{r}+\frac{\partial v_{f}}{\partial r}\right) .
\end{aligned}
$$

We now introduce the following functions $a_{r}$ and $a_{r}$ in the $(r, z)$ plane that vanish at the bubbles equilibrim position $\left(r_{\text {eq }}, z_{\text {coq }}\right)$ :

$$
\begin{aligned}
a_{r}(r, z)= & \frac{6 v}{r_{b}^{2}} u_{f}+2\left(1+C_{a}\right)\left(u_{f} \frac{\partial u_{f}}{\partial r}+w_{f} \frac{\partial u_{f}}{\partial z}-\frac{v_{f}^{2}}{r}\right) \\
& +\frac{2 C_{a}}{r}\left[v_{f}+\frac{r_{b}^{2}\left(1+C_{d}-C_{l}\right)}{3 v} u_{f}\left(\frac{v_{f}}{r}+\frac{\partial v_{f}}{\partial r}\right)\right]^{2} \\
& -2 C_{l} w_{f}\left(\frac{\partial u_{f}}{\partial z}-\frac{\partial w_{f}}{\partial r}\right) \\
& -\frac{2 C_{l}\left(1+C_{a}+C_{l}\right) r_{b}^{2}}{3 v} u_{f}\left(\frac{\partial v_{f}}{\partial r}+\frac{v_{f}}{r}\right)^{2},
\end{aligned}
$$

and

$$
\begin{aligned}
a_{z}(r, z)= & \frac{6 v}{r_{b}^{2}} w_{f}+2\left(1+C_{a}\right)\left(u_{f} \frac{\partial w_{f}}{\partial r}+w_{f} \frac{\partial w_{f}}{\partial z}\right) \\
& +2 C_{f} u_{f}\left(\frac{\partial w_{f}}{\partial z}-\frac{\partial w_{f}}{\partial r}\right)+2 g .
\end{aligned}
$$

The bubble dynamical equations system in the $(r, z)$ plane can be expressed as follows:

$$
\begin{aligned}
& -\frac{6 v}{r_{b}^{2}} u_{b}-2 C_{d} \frac{d u_{b}}{d t}+2 C_{l} w_{b}\left(\frac{\partial u_{f}}{\partial z}-\frac{\partial w_{f}}{\partial r}\right)+a_{r}(r, z)=0, \\
& -\frac{6 v}{r_{b}^{2}} w_{b}-2 C_{d} \frac{d w_{b}}{d t}-2 C_{l} u_{b}\left(\frac{\partial u_{f}}{\partial z}-\frac{\partial w_{f}}{\partial r}\right)+a_{z}(r, z)=0 .
\end{aligned}
$$

Once equilibrim positions have been determined, the stability of the equilibrium can be questioned. For this purpose, the dynamical equations system is made linear around these equilibrium positions. This implies

$$
[A]\left\{\begin{array}{c}
r-r_{\text {eq }} \\
z-z_{\text {eq }} \\
u_{b} \\
w_{b}
\end{array}\right\}=\frac{d}{d t}\left\{\begin{array}{c}
r-r_{\text {eq }} \\
z-z_{\text {eq }} \\
u_{b} \\
w_{b}
\end{array}\right\} \text {, }
$$

with

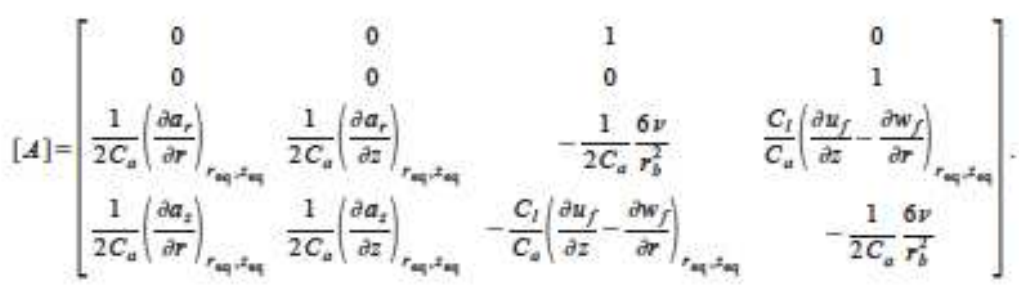

Equilibrium positions are expected to be stable positions, on the condition that the eigenvalues of matrix $A$ bave a negative real part.

'N. H. Thomas, I. R. Authe, K. Seos, and I. C. R. Hunt, "Fntropmest and transport of tubblos by trosient large oddies in multiphase turbeleat shos flown," Procesoding of the Internationsl Confarosce on the Physical Mod-

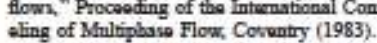

eling of Multiphase Flow, Cosventry (1983). "G. L. Chahine, I. Delopoule, and P. Hasuraert, "Stuty of the interaction Flow ASME 153, 39 (1993)

' $\mathrm{K}$. Sense, J. C. R. Humt, and N. H Theman, The rolo of cobarent stractaros in babble transpert by turbalent shesr flown," J. Fhid Mach 259, 219 (1994).

E. Climent and J. Magnaradet "Modifications d'me cosche de melnige

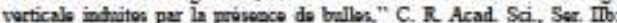
Moc., Phyz., Chim, Astron 326, 627 (1998)

'Y. Shiomi, H. Katrume, K Alagara, and M. Oraura, "Two-phase flow in anmulns with a rotating imer cyirodar ffor porteen in bubbly flow rexiog), "Nacl. Eng. Dor. 141, 27 (1993).

"K. Atbban, I. Footrine, and J-E Wenfreid "Frathy turbulent CocotteTrylor bobbly fow pattems," I. Fhid Mach 422, 55 (2000).

'H. Djocide, I. F. Favi, I. Y. Ballerd, ad D. H. Fruman, "Bubble captem and migration in Couste-Taylor Alorx," Exp. Frids 26, 233 (1999). "G. I. Tryler, "Stability of a viscous liquid contained betwoen two rotating cylindor," Philon. Tran R. Soc. Lomdon, Sar. A 223, 289 (1923). "C. D. Andereck, S. S. Lis and H. L. Swrinay "Flow regimes in a circaly Coostte systam with indopendently rotzing clinders," I. Frid Mech $164,155(1986)$

"M. German and H. L. Swimnoy, "Spatial and tarpornl characteristics of modvlasod waves in the circular Couvets rystem, "I. Frid Mach 117, 123 (1982). 
"T. I. Lim, Y T. Chow, and Q. Xino, "A por flow rogime in a TryiarCouotto flow," Phys Fhids 10, 3233 (1998).

R. E. A. Andt and A. P. Keller, "Whar quality offects on critation inception in a trailing vortox," I. Fluids Feng 114, 430 (1992).

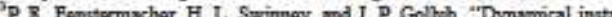

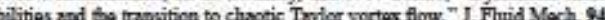
$103(1979)$

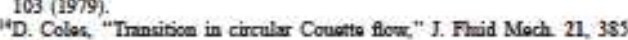
D. Color

R. S. Sherw, C. D. Andoreck, L. A. Reith and H. L. Suinner, "Superpouition of traveling warven in the circular Couetos system," Plyz. Rov. Iet

48, 1172 (1952). dern," J. Firid Mach. 14, 336 (1962).

J. P. Gollub and M. H Freilich "Optical hetarodyne tost of perturtation arpantions for the Trylor inatability," Phys. Fluids 19, 618 (1976).

J. Magnesodot, M. Rivero, and I. Fabre, "Acceleratod flown port a rigit sphere ar a spharical bebbla. I: Steody straining florx," J. Fluid Mech. 284, 97 (1995).

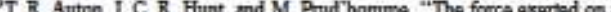
boty in inviscid mbtoady nommiform rotational flow," J. Fhid Moch
197, 241 (1988)

I. Magusudet and D. Logeodro, "Some aspocts of the lift force ca a sqbarical bubble, Appl Sci. Kor. 58, 441 (1998)

${ }^{2}$ D. Legente and J. Mazomidot, "The lift force on a spherical bubblo in a viscous lious sherr flow," J. Fluid Mach. 368, 81 (1998)

${ }^{n}$ Y. Reasidy mad D. Joseph "Cosats flow of two fluids between concentric givinan," I. Fhid Mech. 150, 381 (1985)

S. Gopalan and J. Kath, "EFfoct of astrained bubbles on the strocture of sortor ringa," J. Fuid Mach. 393, 171 (1999).

"S. I Wurcley and R. Lueptor, "Avimithal volocity in neparcritical circuIar Couvte flore," Exp. Fhads 18, 1 (1994).

$\mho_{1}$. L. Maris, "Modeling of the shim friction and heat trantor in turbulont two-componeant babbly flown in pipes," Int. I. Maltiphase Flow 13, 309 (1987).

"C. Gabillat, C. Colin, and J. Fatur, "Experimental atady of babbla injoction in a tutulent boundary layer," Int J. Mfultiphase Flow 28, 553 (2002).

${ }^{n}$ H Djeridi, C. Gatollet, and J. Y. Billand, "Ecoudansent do Couotto Tryibe Diphasique," C. R. Acad Sai. Paria, Sar Ib. Mac., Plyz., Chim., Astron 330. 113 (2002). 